Bull. Soc. math. France

131 (4), 2003, p. 603-616

\title{
THE ACTION SPECTRUM NEAR POSITIVE DEFINITE INVARIANT TORI
}

\author{
BY PATRICK BERNARD
}

\begin{abstract}
We show that the Birkhoff normal form near a positive definite KAM torus is given by the function $\alpha$ of Mather. This observation is due to Siburg [Si2], [Si1] in dimension 2. It clarifies the link between the Birkhoff invariants and the action spectrum near the torus. Our extension to high dimension is made possible by a simplification of the proof given in [Si2].

RÉSUMÉ (Le spectre d'action au voisinage des tores invariants à torsion définie)

On montre que la forme normale de Birkhoff au voisinage d'un tore KAM à torsion définie est donnée par la fonction $\alpha$ de Mather. Cette observation est due à Siburg [Si2], [Si1], en dimension 2. Elle clarifie le lien entre les coefficients de Birkhoff et le spectre d'action au voisinage du tore. Notre extension à la dimension supérieure est rendue possible par une simplification de la preuve donnée dans [Si2].
\end{abstract}

\section{Introduction}

Let us consider a smooth symplectic manifold $(\mathcal{M}, \Omega)$ of dimension $2 n$, and a $C^{1}$ symplectic diffeomorphism $\phi: \mathcal{M} \rightarrow \mathcal{M}$. We are going to study the dynamic in the neighborhood of some invariant tori of $\phi$.

Texte reçu le 6 mai 2002, révisé le 22 novembre 2002

Patrick Bernard, EPFL, Switzerland - Institut Fourier, BP 74, 38402 Saint Martin d'Hères Cedex (France) • E-mail : Patrick.Bernard@ujf-grenoble.fr

Url : http://www-fourier.ujf-grenoble.fr/ pbernard

2000 Mathematics Subject Classification. - 37J40, 37J50.

Key words and phrases. - Lagrangian systems, Aubry-Mather theory, Minimizing orbits, Averaged action, Invariant torus, Normal forms, Action spectrum.

BULlETIN DE LA SOCIÉTÉ MATHÉMATIQUe DE FRANCE

(C) Société Mathématique de France

$0037-9484 / 2003 / 603 / \$ 5.00$ 
1.1. We note $\mathbb{T}=\mathbb{R} / \mathbb{Z}$. We use the sign + for sums in $\mathbb{R}^{n}$ or $\mathbb{T}^{n}$, and we also define $q+v=q+v \bmod \mathbb{Z}^{n} \in \mathbb{T}^{n}$ in the standard way when $q \in \mathbb{T}^{n}$ and $v \in \mathbb{R}^{n}$. We identify $T^{*} \mathbb{T}^{n}$, endowed with its standard symplectic structure, with the product $\mathbb{T}^{n} \times \mathbb{R}^{n}$, and use coordinates $(q, p) \in \mathbb{T}^{n} \times \mathbb{R}^{n}$. We note $\mathbb{T}_{0}^{n}$ the zero section of $T^{*} \mathbb{T}^{n}$, that is the submanifold $\mathbb{T}^{n} \times\{0\}$ of $\mathbb{T}^{n} \times \mathbb{R}^{n}$. In the following, we say that a neighborhood of $\mathbb{T}_{0}^{n}$ in $\mathbb{T}^{n} \times \mathbb{R}^{n}$ is simple if it is fiberwise convex, that is if its intersection with any fiber $q \times \mathbb{R}^{n}$ is convex. A local chart of an invariant torus $\mathcal{T}$ is a symplectic diffeomorphism from a simple neighborhood of $\mathbb{T}_{0}^{n}$ onto its image in $\mathcal{M}$ whose restriction to $\mathbb{T}_{0}^{n}$ is a diffeomorphism onto $\mathcal{T}$. Given a torus $\mathcal{T}$, we call simple neighborhood of $\mathcal{T}$ the image of a local chart. There exist local charts of $\mathcal{T}$ if and only if $\mathcal{T}$ is Lagrangian. We identify the vector spaces $H^{1}\left(\mathbb{T}^{n}, \mathbb{R}\right)$ and $H_{1}\left(\mathbb{T}^{n}, \mathbb{R}\right)$ with $\mathbb{R}^{n}$.

1.2. Definition. - An invariant torus $\mathcal{T}$ is called a $C^{k}$ positive definite quasiperiodic invariant torus if there exists a $C^{k}$ local chart $\tau$ of $\mathcal{T}$, a vector $\omega \in \mathbb{R}^{n}$ and a positive definite matrix $A$ such that, as $p \rightarrow 0$,

$$
\tau^{-1} \circ \phi \circ \tau(q, p)=(q+\omega+A p, p)+o(p) .
$$

1.3. It follows from the definition above that the torus $\mathcal{T}$ is a Lagrangian invariant torus on which the dynamic is conjugated to a translation. In a simple neighborhood of a quasi-periodic invariant torus $\mathcal{T}$, the diffeomorphism $\phi$ is homotopic to the identity. It is convenient to choose once and for all a simple neighborhood $U_{0}$ of $\mathcal{T}$ and a homotopy $\phi_{t}$ between the identity and $\phi_{\mid U_{0}}$. We can assume that $\phi_{t}(\mathcal{T})=\mathcal{T}$ for each $t$. Let us fix a smaller simple neighborhood $U_{1}$ such that $\phi_{t}(x) \in U_{0}$ for all $x \in U_{1}$ and $t \in[0,1]$. A periodic orbit $X=\left(x_{0}, x_{1}, \ldots, x_{T}=x_{0}\right)$ of $\phi$ contained in $U_{1}$ has a well defined homology $[X] \in H_{1}(\mathcal{T}, \mathbb{Z})$. This homology is defined by extending the periodic orbit to a periodic curve in $U_{0}$ using the homotopy $\phi_{t}$, and by identifying $H_{1}\left(U_{0}, \mathbb{Z}\right)$ with $H_{1}(\mathcal{T}, \mathbb{Z})$.

1.4. Definition. - We say that the invariant torus $\mathcal{T}$ has a Birkhoff normal form of order $k$ if there exists a local chart $\tau$ of $\mathcal{T}$ such that

$$
\tau^{-1} \circ \phi \circ \tau(q, p)=\left(q+\mathrm{d} h_{k}(p), p\right)+o_{k-1}(p),
$$

where $h_{k}: \mathbb{R}^{n} \rightarrow \mathbb{R}$ is polynomial of degree $k$ satisfying $h_{k}(0)=0$. Such a polynomial is called a Birkhoff normal form of degree $k$ near $\mathcal{T}$.

Let us mention the following result which should be seen as a motivation for the definitions above (see [La]).

1.5. Proposition. - Let $\phi$ be a smooth symplectomorphism on $\mathcal{M}$, and let $\mathcal{T}$ be a smooth invariant torus. Assume that the dynamic on $\mathcal{T}$ is conjugated to a Diophantine translation, and that $\mathcal{T}$ is Lagrangian (this hypothesis is automatic if $\Omega$ is exact). Then $\mathcal{T}$ admits Birkhoff normal forms to all orders. 
1.6. The restriction of $\Omega$ to $U_{0}$ is exact. A local Liouville form is a one form $\lambda$ on $U_{0}$ such that $\mathrm{d} \lambda=\Omega$. There exists a local Liouville form $\lambda$ whose restriction to $\mathcal{T}$ is zero. All the Liouville forms with this property differ by the differential of a function $f$ which is null on $\mathcal{T}$. If $\lambda$ is such a Liouville form, there exists a unique function $g$ on $U_{0}$ such that $\mathrm{d} g=\phi^{*} \lambda-\lambda$ and $\left.g\right|_{\mathcal{T}}=0$. If $\lambda^{\prime}=\lambda+\mathrm{d} f$ is another Liouville form with the same property, then the associated function is $g^{\prime}=g+f \circ \phi-f$.

1.7. Let $X=\left(x_{0}, x_{1}, \ldots, x_{T}=x_{0}\right)$ be a periodic orbit. We define its action

$$
\mathcal{A}(X)=\sum_{i=1}^{T} g\left(x_{i}\right)
$$

it is easy to see that this sum does not depend of the choice of the Liouville form $\lambda$ whose restriction to $\mathcal{T}$ is zero used to define $g$.

1.8. A periodic orbit $X$ contained in the simple neighborhood $U_{1}$, see 1.3 , has a period $T(X) \in \mathbb{Z}$, an homology (or rotation number) $[X] \in H_{1}(\mathcal{T}, \mathbb{Z})$ and an action $\mathcal{A}(X)$. For each simple neighborhood $U$ contained in $U_{1}$, let us define the labelled $U$-action spectrum as the set

$$
A_{U}=\{(\mathcal{A}(X), T(X),[X])\} \subset \mathbb{R} \times \mathbb{Z} \times H_{1}(\mathcal{T}, \mathbb{Z}),
$$

where $X$ ranges over periodic orbits contained in $U$. For each cohomology class $c \in H^{1}(\mathcal{T}, \mathbb{R})$, define

$$
\alpha_{U}(c)=\sup _{(a, T, w) \in A_{U}} \frac{c w-a}{T} .
$$

1.9. THEOREM. - Let us consider a $C^{1}$ symplectic diffeomorphism $\phi$ and a $C^{1}$ positive definite quasi-periodic invariant torus $\mathcal{T}$. Let us fix a homotpy between $\phi$ and the identity in a simple neighborhood $U_{0}$ of $\mathcal{T}$. For each sufficiently small simple neighborhood $U$, the function $\alpha_{U}$ is finite and convex in a neighborhood of 0 . The germ at $c=0 \in H^{1}(\mathcal{T}, \mathbb{R})$ of the function $\alpha_{U}$ does not depend on $U$.

We call $\alpha$ this common germ. We have defined a germ of function

$$
\alpha: H^{1}(\mathcal{T}, \mathbb{R}) \longrightarrow \mathbb{R}
$$

which is a symplectic invariant and depends only on the local labelled action spectrum. Let us call it the averaged energy. This function has been introduced and studied via a slightly different definition by Mather in [Ma], where Theorem 1.9 is already present. We shall present a simple and self contained proof of this result. Note that the finiteness of $\alpha(0)$ is already a non trivial fact which implies the existence of periodic orbits in any neighborhood of $\mathcal{T}$. Our main point here is the relation between the action spectrum, the averaged energy $\alpha$ and the Birkhoff normal forms. 
1.10. TheOREM. - In the setting of Theorem 1.9, assume in addition that the torus $\mathcal{T}$ has a Birkhoff normal form $h_{k}$ of order $k$. We have

$$
\alpha=h_{k} \circ \tau^{*}+o_{k}
$$

where $\tau^{*}: H^{1}(\mathcal{T}, \mathbb{R}) \longrightarrow H^{1}\left(\mathbb{T}_{0}^{n}, \mathbb{R}\right)=\mathbb{R}^{n}$ is the mapping associated to $\tau$.

1.11. The function $\alpha$ depends on the choice of the homotopy between the identity and $\phi$. If another homotopy had been chosen, the associated function $\tilde{\alpha}$ would satisfy

$$
\tilde{\alpha}(c)=\alpha(c)+\langle\xi, c\rangle
$$

for some $\xi \in H_{1}(\mathcal{T}, \mathbb{Z})$.

1.12. As a consequence of Theorem 1.10, we obtain a unicity result for Birkhoff normal forms of positive definite tori. Once a homotopy to the identity has been chosen, the normal form is well defined as a polynomial on $H^{1}(\mathcal{T}, \mathbb{R})$. As a consequence, when seen as a polynomial on $\mathbb{R}^{n}$, the Birkhoff normal form is well defined only up to the action of $\mathrm{Gl}_{n}(\mathbb{Z})$.

1.13. It is a straightforward consequence of Theorem 1.10 that the Birkhoff normal form of order $k$, provided it exists, depends only on the local labelled action spectrum. Recall Proposition 1.5.

1.14. We discuss some sufficient conditions for a quasi-periodic invariant torus to be positive definite in Section 2. We then clarify some conventions concerning the choice of the homotopy $\phi_{t}$ and of the rotation vector $\omega$ in Section 3. The remaining of the paper is devoted to the proof of the two theorems above.

1.15. We work in coordinates $\mathbb{T}^{n} \times \mathbb{R}^{n}$, and first introduce the generating function of our diffeomorphism in Section 4. This generating function is used to define a new action, which is closely connected to the action defined in 1.7 above. In Section 5, we define a new averaged energy using the new action. The links between this new averaged energy and the Birkhoof normal forms are easily established using a property of monotony. This observation is our first improvement compared with the proof of [Si2]. We are then reduced to observe that the two averaged energies coincide. One of the important points is that the germ of the averaged energy is local, i.e. depends only of the germ of $\phi$ along the invariant torus. A similar property was proved in [Si2] using the existence of KAM circles around the elliptic fixed point. It is our second improvement to give a purely variational proof independent of KAM theory. This allows to extend the result to higher dimension where KAM tori do not confine the dynamic. The main step is the existence of a family of periodic orbits near the invariant torus. This property is proved in Section 6. Note that the convexity of the averaged action $\alpha$ is a direct consequence of its definition as a supremum of linear functions.

TOME $131-2003-\mathrm{N}^{\mathrm{O}} 4$ 


\section{Positive definite KAM tori}

The definition of a positive definite quasi-periodic invariant torus given in 1.2 is not intrinsic and requires some comments. Let us consider a smooth symplectic diffeomorphism $\phi$ and a smooth invariant torus $\mathcal{T}$. We assume that the induced dynamic $\phi_{\mid \mathcal{T}}$ is conjugated to a Diophantine translation. In addition, we assume that the torus $\mathcal{T}$ is Lagrangian. This hypothesis is automatic if the symplectic form $\Omega$ is exact, in view of the following remark of M. Herman (see [He]):

2.1. Let $\phi$ be a diffeomorphism of an exact symplectic manifold $(\mathcal{M}, \Omega=\mathrm{d} \lambda)$. Let $\mathcal{T}$ be an invariant torus with an ergodic linear induced dynamic. Then the torus $\mathcal{T}$ is Lagrangian. To see this, let us consider an embedding $\eta: \mathbb{T}^{n} \rightarrow \mathcal{M}$ of image $\mathcal{T}$ and such that $R=\eta_{\mid \mathcal{T}}^{-1} \circ \phi \circ \eta$ is a Diophantine translation. Calling $\mu=\eta^{*} \lambda$ the restriction of the Liouville form, we have $\mathrm{d}\left(R^{*} \mu-\mu\right)=0$. Now writing $\mu=a(q) \mathrm{d} q$, we obtain that $a \circ R=a$, this implies that $a$ is constant since $R$ is an ergodic translation. As a consequence, $d \mu=0$, which means that $\mathcal{T}$ is Lagrangian since $\mathrm{d} \mu=\eta^{*} \Omega$.

2.2. Since $\mathcal{T}$ is Lagrangian, there exist local charts, this results from a celebrated theorem of Weinstein, see for exemple [DS]. Let $\tau$ be a local chart of $\mathcal{T}$ such that $\tau^{-1} \circ \phi_{\mid \mathcal{T}} \circ \tau_{\mid \mathbb{T}_{0}^{n}}$ is a translation. Then there exist a vector $\omega \in \mathbb{R}^{n}$ and a smooth function $A: \mathbb{T}^{n} \longrightarrow \mathcal{S}_{n}$ of symmetric matrices such that

$$
\tau^{-1} \circ \phi \circ \tau(q, p)=(q+\omega+A(q) p, p)+o(p) .
$$

In order to see this, note that $\tau^{-1} \circ \phi \circ \tau(q, p)=(q+\omega+A(q) p, B(q) p)+o(p)$, with two function $A, B: \mathbb{T}^{n} \rightarrow M_{n}$ of matrices. It is then straightforward to check that this mapping is symplectic if and only if $B(q)=\operatorname{Id}$ and $A(q)$ is symmetric for each $q$.

2.3. It is known that there exists a new chart $\tilde{\tau}$ such that

$$
\tilde{\tau}^{-1} \circ \phi \circ \tilde{\tau}(q, p)=(q+\omega+A p, p)+o(p) .
$$

where the matrix $A$ is the average

$$
A=\int_{\mathbb{T}^{n}} A(q)
$$

with respect to the Haar measure. The chart $\tilde{\tau}$ is obtained by composing $\tau$ with an averaging transformation. More precisely, let us consider the time- 1 flow $\psi$ of the Hamiltonian $H(q, p)=\frac{1}{2}\langle a(q) p, p\rangle$, where $a(q)$ is a smooth function of symmetric matrices. We have

$$
\psi(q, p)=(q+a(q) p, p)+o(p) .
$$

BULLETIN DE LA SOCIÉTÉ MATHÉMATIQUE DE FRANCE 
A calculation shows that the chart $\tilde{\tau}=\tau \circ \psi$ has the desired property provided that $a(q)$ solves the equation

$$
a(q)-a(q+\omega)=A(q)-A .
$$

It is known that this equation has a smooth solution if $\omega$ is Diophantine and if $A=\int A(q)$.

2.4. Lemma. - The torus $\mathcal{T}$ is positive definite if and only if the averaged matrix $A$ is positive definite in any local chart $\tau$ such that $\tau^{-1} \circ \phi_{\mid \mathcal{T}} \circ \tau_{\mid \mathbb{T}_{0}^{n}}$ is a translation.

Proof. - It is clear in view of the remarks above that $\mathcal{T}$ is positive definite if the averaged matrix $A$ is positive definite in any local chart. Conversely, let us consider a positive definite torus $\mathcal{T}$ and let $\tau$ be the local chart given by Definition 1.2. Let $\tau^{\prime}$ be another local chart such that $\tau^{\prime-1} \circ \phi_{\mid \mathcal{T}} \circ \tau_{\mid \mathbb{T}_{0}^{n}}^{n}$ is a translation, and let $\tilde{\tau}^{\prime}$ be the associated chart given by averaging. We have

$$
\tilde{\tau}^{\prime-1} \circ \phi \circ \tilde{\tau}^{\prime}(q, p)=\left(q+\omega^{\prime}+A^{\prime} p, p\right)+o(p),
$$

where $A^{\prime}$ is a symmetric matrix. The matrix $A^{\prime}$ is positive definite because there exists a matrix $a \in \mathrm{Gl}_{n}(\mathbb{R})$ such that $A^{\prime}={ }^{t} a A a$. This is the result of a simple computation based on the fact that the differential of $\tau^{-1} \tilde{\tau}^{\prime}$ at any point of the torus can be written in block forms as

$$
\left(\begin{array}{cc}
a^{-1} & b a \\
0 & a
\end{array}\right)
$$

where $b$ is a symmetric matrix.

\section{Rotation vector}

We now discuss some questions of definiteness about the rotation vector of quasi-periodic tori. Let us consider a positive definite quasi-periodic invariant torus $\mathcal{T}$, let $\tau: V_{0} \longrightarrow \mathcal{M}$ be a local chart as given by Definition 1.2, and let $U_{0}$ be its image. Assume that a homotopy $\phi_{t}$ of mappings from $U_{0}$ to $\mathcal{M}$ has been chosen such that $\phi_{0}=I d$ and $\phi_{1}=\phi$, and that it satisfies $\phi_{t}(\mathcal{T})=\mathcal{T}$ for each $t$. Consider a simple neighborhood $U_{1}$ such that $\phi_{t}\left(U_{1}\right) \subset U_{0}$ for each $t$.

3.1. The rotation vector of $\phi_{\mid \mathcal{T}}$ is an element $\xi$ of $H_{1}(\mathcal{T}, \mathbb{R})$ defined as follows. Since $\phi_{\mid \mathcal{T}}$ is conjugated to a translation, there exists a sequence $T_{n}$ of integers such that

$$
\psi_{n}=\left(\phi_{\mid \mathcal{T}}\right)^{T_{n}} \longrightarrow \mathrm{Id} .
$$

Let us fix a point $x \in \mathcal{T}$, and let $D$ be a simply connected neighborhood of $x$. When $n$ is large enough, the point $\psi_{n}(x)$ is in $D$. There is a path between $x$ 
and $\psi_{n}(x)$ given by the homotopy $\phi_{t}$. We can close this path by a path in $U$. The homology $w_{n} \in H_{1}(\mathcal{T}, \mathbb{Z})$ of the obtained loop is well-defined and we set

$$
\xi=\lim \frac{w_{n}}{T_{n}}
$$

Let $\eta: \mathbb{T}^{n} \rightarrow \mathcal{T}$ be a diffeomorphism. Identifying $H_{1}\left(\mathbb{T}^{n}, \mathbb{R}\right)$ with $\mathbb{R}^{n}$, we have

$$
\eta^{-1} \circ \phi_{\mid \mathcal{T}} \circ \eta(q)=q+\eta_{*}^{-1}(\xi)
$$

3.2. The vector $\omega$ in Definition 1.2 is defined only modulo $\mathbb{Z}^{n}$. We shall assume for definiteness that the following compatibility condition holds between the choice of the homotopy $\phi_{t}$ (and hence of the rotation vector $\xi$ ) and the choice of the rotation vector $\omega$ :

$$
\xi=\tau_{*}(\omega),
$$

where $\omega$ is seen as an element of $H_{1}\left(\mathbb{T}_{0}^{n}, \mathbb{R}\right)=\mathbb{R}^{n}$.

3.3. Let $Q(q, p): \mathbb{T}^{n} \times \mathbb{R}^{n} \rightarrow \mathbb{T}^{n}$ be the first component of $\tau^{-1} \circ \phi \circ \tau(q, p)$. Let $V(q, p): \mathbb{T}^{n} \times \mathbb{R}^{n} \rightarrow \mathbb{R}^{n}$ be the unique continuous function such that $V(q, 0)=\omega$ and $Q(q, p)=q+V(q, p)$. Let us consider a periodic orbit $X=\left(x_{0}, x_{1}, \ldots, x_{T}=x_{0}\right)$ of $\phi$ contained in $U_{1}$, and set $\left(q_{i}, p_{i}\right)=\tau^{-1}\left(x_{i}\right)$. Assuming that the homotopy $\phi_{t}$ and the rotation vector $\omega$ satisfy the compatibility condition 3.2 , we have

$$
[X]=\tau_{*}\left(\sum_{i=0}^{T-1} V\left(q_{i}, p_{i}\right)\right) .
$$

This can be seen by considering the unique lifting $F_{t}$ of $\tau^{-1} \circ \phi_{t \mid U_{0}} \circ \tau$ to the universal cover $\tilde{V}_{0} \subset \mathbb{R}^{n} \times \mathbb{R}^{n}$ of $V_{0}$ satisfying $F_{0}=\mathrm{Id}$. Let us note $F=F_{1}$ the lifting of $\tau^{-1} \circ \phi_{\mid U_{0}} \circ \tau$ obtained in this way. We have

$$
F(q, p)=\left(q+V\left(q \bmod \mathbb{Z}^{n}, p\right), .\right)
$$

and the homology of $X$ is clearly $\tau_{*}\left(\tilde{q}_{T}-\tilde{q}_{0}\right)$, where $\left(\tilde{q}_{i}, \tilde{p}_{i}\right)=F^{i}\left(\tilde{q}_{0}, \tilde{p}_{0}\right)$ is a lifting of $\left(q_{i}, p_{i}\right)$.

\section{The generating function and the action}

In this section, we introduce the tools necessary to study the periodic orbits of $\phi$ near $\mathcal{T}$. We work in coordinates $\mathbb{T}^{n} \times \mathbb{R}^{n}$.

BULLETIN DE LA SOCIÉTÉ MATHÉMATIQUE DE FRANCE 
4.1. Let $\mathcal{S}_{\omega}$ be the set of functions of $C^{2}\left(\mathbb{T}^{n} \times \mathbb{R}^{n}, \mathbb{R}\right)$ with the following properties:

- There exist real numbers $b>a>0$ such that for all $(q, v) \in \mathbb{T}^{n} \times \mathbb{R}^{n}$

$$
a\|v-\omega\|^{2} \leqslant S(q, v) \leqslant b\|v-\omega\|^{2} .
$$

- There exists a positive number $K$ such that $S(q, v)=a\|v-\omega\|^{2}$ when $\|v\| \geqslant K$.

- The mapping $(q, v) \mapsto\left(q, \partial_{2} S(q, v)-\partial_{1} S(q, v)\right)$ is a diffeomorphism of $\mathbb{T}^{n} \times \mathbb{R}^{n}$. We call $G^{S}$, and often $G$, its inverse.

4.2. It is known that to any function $S \in \mathcal{S}_{\omega}$, we can associate the symplectic diffeomorphism $F$ of $\mathbb{T}^{n} \times \mathbb{R}^{n}$ whose graph in $\left(\mathbb{T}^{n} \times \mathbb{R}^{n}\right)^{2}$ is the image of the embedding

$$
\begin{aligned}
\mathbb{T}^{n} \times \mathbb{R}^{n} & \longrightarrow \mathbb{T}^{n} \times \mathbb{R}^{n} \times \mathbb{T}^{n} \times \mathbb{R}^{n}, \\
(q, v) & \longmapsto\left(q, \partial_{2} S(q, v)-\partial_{1} S(q, v), q+v, \partial_{2} S(q, v)\right) .
\end{aligned}
$$

We then have

$$
F^{*} \lambda_{0}-\lambda_{0}=\mathrm{d}\left(S \circ G^{S}\right),
$$

where $\lambda_{0}$ is the canonical form $p \mathrm{~d} q$ of $T^{*} \mathbb{T}^{n}=\mathbb{T}^{n} \times \mathbb{R}^{n}$. The correspondence $S \mapsto F$ between $\mathcal{S}_{\omega}$ and the set $\mathcal{P}_{\omega}$ of mappings that can be obtained this way is bijective. We say that $S$ is the generating function of $F$, or that $F$ is generated by $S$.

4.3. Remark. - It is more classical (see [DS] or [Be]) to define generating functions $\tilde{S}\left(x, x^{\prime}\right): \mathbb{R}^{n} \times \mathbb{R}^{n} \rightarrow \mathbb{R}$. Here we have used different coordinates

$$
\tilde{S}\left(x, x^{\prime}\right)=S\left(x \bmod \mathbb{Z}^{n}, x^{\prime}-x\right) .
$$

4.4. In order to work on the configuration space $\mathbb{T}^{n}$, let us define, for each $e \in \mathbb{R}^{n}$, the function $H_{e}: \mathbb{T}^{n} \times \mathbb{T}^{n} \rightarrow \mathbb{R}$ by

$$
H_{e}\left(q, q^{\prime}\right)=\min _{q+v=q^{\prime}} S(q, v)-e v .
$$

It is not hard to see that the function $H_{e}$ is Lipschitz for all $e$ (see [Be] for example). We define the action of a configuration $\left(q_{0}, \ldots, q_{T}\right) \in\left(\mathbb{T}^{n}\right)^{T+1}$ by

$$
H_{e}^{T}\left(q_{0}, \ldots, q_{T}\right)=\sum_{i=0}^{T-1} H_{e}\left(q_{i}, q_{i+1}\right) .
$$

We will pay a special attention to periodic orbits, and define the space

$$
E^{T}=\left\{\left(q_{0}, \ldots, q_{T}\right) \in\left(\mathbb{T}^{n}\right)^{T+1} \text { such that } q_{T}=q_{0}\right\}
$$

of $T$-periodic configurations. 
4.5. Definition. - An orbits segment $\left(q_{i}, p_{i}\right)=F^{i}\left(q_{0}, p_{0}\right), 0 \leqslant i \leqslant T$ is said e-minimal if

$$
S\left(q_{i}, V\left(q_{i}, p_{i}\right)\right)-e V\left(q_{i}, p_{i}\right)=H_{e}\left(q_{i}, q_{i+1}\right)
$$

for each $i$.

4.6. LEMmA. - If $\left(q_{0}, \ldots, q_{T}\right) \in E^{T}$ is minimizing $H_{e}^{T}$ for some $e$, then there exists a sequence $p_{0}, \ldots, p_{T}=p_{0}$ such that $\left(q_{0}, p_{0}\right),\left(q_{1}, p_{1}\right), \ldots,\left(q_{T}, p_{T}\right)$ is a $T$-periodic orbit of $F$ and is e-minimal.

Proof. - Let $\left(\bar{q}_{0}, \ldots, \bar{q}_{T}\right) \in E^{T}$ be a configuration minimizing $H_{e}^{T}$. Let us consider the set $\mathcal{E}^{T}$ of sequences

$$
\left(\left(q_{0}, v_{0}\right), \ldots,\left(q_{T}, v_{T}\right)\right) \subset\left(\mathbb{T}^{n} \times \mathbb{R}^{n}\right)^{T+1}
$$

such that $q_{i+1}=q_{i}+v_{i}$ and $\left(q_{T}, v_{T}\right)=\left(q_{0}, v_{0}\right)$. Let us define the action $\Sigma$ on $\mathcal{E}^{T}$ by

$$
\Sigma\left(\left(q_{0}, v_{0}\right), \ldots,\left(q_{T}, v_{T}\right)\right)=\sum_{i=0}^{T-1} S\left(q_{i}, v_{i}\right)-e v_{i} .
$$

Its minimum is $H_{e}^{T}\left(\bar{q}_{0}, \ldots, \bar{q}_{T}\right)$ an there exists $\left(\bar{v}_{0}, \ldots, \bar{v}_{T}\right) \in\left(\mathbb{R}^{n}\right)^{T+1}$ such that $\left(\bar{q}_{i}, \bar{v}_{i}\right)$ is minimizing $\Sigma$. It is not hard to see that the sequence $\left(\bar{q}_{i}, \bar{p}_{i}\right)$, where

$$
\bar{p}_{i}=\partial_{2} S\left(\bar{q}_{i}, \bar{v}_{i}\right)-\partial_{1} S\left(\bar{q}_{i}, \bar{v}_{i}\right)=\partial_{1} S\left(\bar{q}_{i-1}, \bar{v}_{i-1}\right)
$$

is a $T$-periodic orbit of $F$ and is $e$-minimal.

4.7. A digression via Legendre transformation may be useful. Let $\ell: \mathbb{R}^{n} \rightarrow \mathbb{R}$ be a strongly convex function, that is a $C^{2}$ proper function with uniformly positive definite Hessian. We define its Legendre transform $\ell^{*}: \mathbb{R}^{n} \rightarrow \mathbb{R}$ by

$$
\ell^{*}(p)=\sup _{v \in \mathbb{R}} p v-\ell(v)
$$

which is a $C^{2}$ convex function. The correspondence $\ell \mapsto \ell^{*}$ is non increasing. Here is an equivalent definition. The mapping $v \mapsto \mathrm{d} \ell(v)$ is a diffeomorphism $\mathcal{L}$ of $\mathbb{R}^{n}$ (we identify $\mathbb{R}^{n}$ with its dual). We have $\ell^{*}(p)=p \mathcal{L}^{-1}(p)-\ell\left(\mathcal{L}^{-1}(p)\right.$ ). It is well known that $\ell^{* *}=\ell$, and that we have

$$
p=\mathrm{d} \ell(v) \Longleftrightarrow v=\mathrm{d} \ell^{*}(p),
$$

that is, if $\mathcal{L}^{*}$ is the diffeomorphism $p \mapsto \mathrm{d} \ell^{*}(p)$, then $\mathcal{L}^{*}=\mathcal{L}^{-1}$. It is not hard to see that if $\ell=\ell_{k}+o_{k}$, then $\ell^{*}=\ell_{k}^{*}+o_{k}$.

BULlEtiN DE LA SOCIÉtÉ MATHÉMATIQUE DE FRANCE 
4.8. If $\mathcal{T}$ is a positive definite KAM torus, there exists a local chart $\tau: V_{0} \rightarrow$ $\mathcal{M}$ of $\mathcal{T}$ and a diffeomorphism $F \in \mathcal{P}_{\omega}$ such that $F_{\mid V_{0}} \circ \tau^{-1}=\tau^{-1} \circ \phi$. In addition, if $\mathcal{T}$ has a Birkhoff normal form $h_{k}$ of order $k$, then we can chose $F$ such that its generating function $S$ satisfies

$$
S(q, v)=\ell_{k}(v)+o_{k}(v),
$$

where $\ell_{k}$ is a convex function whose Legendre transform is equal to $h_{k}$ in a neighborhood of 0 . All this follows from the fact that the diffeomorphism $F$ generated by $S_{k}(q, v)=\ell_{k}(v)$ is $(q, p) \mapsto F(q, p)=\left(q+\mathrm{d} h_{k}(p), p\right)$. Note in addition that we have $\ell_{k}(\omega)=-h_{k}(\omega)=0$ since $\mathrm{d} h_{k}(0)=\omega$.

4.9. Let $U_{1}$ be the simple neighborhood defined in 1.3, and let $V_{1}=\tau^{-1}\left(U_{1}\right)$. There is a one to one correspondence between the orbits of $\phi$ contained in $U_{1}$ and the orbits of $F$ contained in $V_{1}$. Let $X$ be a $T$-periodic orbit of $\phi$ in $U_{1}$, and $Y$ the corresponding orbit of $F$. The actions we have defined for $X$ and $Y$ are closely connected.

4.10. Lemma. - Setting $e=\tau^{*} c$, we have

$$
\mathcal{A}(X)-c[X] \geqslant H_{e}^{T}(Y)
$$

with equality if and only if $Y$ is e-minimal.

Proof. - Let us note $\lambda_{0}$ the canonical Liouville form $p \mathrm{~d} q$ of $T^{*} \mathbb{T}^{n}=\mathbb{T}^{n} \times \mathbb{R}^{n}$. We can use the Liouville form $\lambda=\tau^{*-1} \lambda_{0}$ to compute the function $g$ involved in the action $\mathcal{A}(Y)$. We have

$$
\tau^{*}(d g)=F^{*} \lambda_{0}-\lambda_{0}=\mathrm{d}\left(S \circ G^{S}\right) .
$$

Hence $g \circ \tau=S \circ G^{S}$. Let $\left(q_{i}, p_{i}\right)=F^{i}\left(q_{0}, p_{0}\right)$ be the points of the orbit $Y$. We have

$$
g \circ \tau\left(q_{i}, p_{i}\right)=S\left(q_{i}, V\left(q_{i}, p_{i}\right)\right) \quad \text { and } \quad q_{i}+V\left(q_{i}, p_{i}\right)=q_{i+1} .
$$

It follows that

$\mathcal{A}(X)-c[X]=\sum_{i=0}^{T-1} S\left(q_{i}, V\left(q_{i}, p_{i}\right)\right)-e V\left(q_{i}, p_{i}\right) \geqslant H_{e}^{T}\left(q_{0}, q_{1}, \ldots q_{T}\right)=H_{e}^{T}(Y)$,

with equality if and only if $S\left(q_{i}, V\left(q_{i}, p_{i}\right)\right)-e V\left(q_{i}, p_{i}\right)=H_{e}\left(q_{i}, q_{i+1}\right)$ for each $i$.

\section{The averaged energy}

In the present section, we introduce the averaged energy $\alpha^{S}$ associated to a generating function $S \in \mathcal{S}$. We prove that this function is equal to the function $\alpha_{U}$ and use it to prove the main properties of $\alpha$. 
5.1. Let us define

$$
m^{T}(e)=\min _{E^{T}} H_{e}^{T} \quad \text { and } \quad \alpha^{S}(e)=-\inf _{T \geqslant 0}\left(\frac{m^{T}(e)}{T}\right) .
$$

5.2. Proposition. - The correspondence $S \mapsto \alpha^{S}$ is non increasing.

This remark is elementary, but deserves attention as a new observation. It enables a neat simplification of the proofs compared with [Si2]. It is a standard in the calculus of variations to exploit the monotonicity of certain critical levels. Here we mean that if $S^{\prime} \in \mathcal{S}$ is another generating function such that $S^{\prime}(q, v) \geqslant S(q, v)$ for each $(q, v)$, then the associated functionals $H_{e}^{T}$ and $H_{e}^{\prime T}$ satisfy $H_{e}^{\prime T}(Q) \geqslant H_{e}^{T}(Q)$ for each $T$, $e$, and $Q$. Hence $\alpha^{S^{\prime}}(e) \leqslant \alpha^{S}(e)$ for each $e$. The proof is just a consequence of the fact that

$$
-\alpha^{S}(e)=\min \frac{1}{T} \sum_{i=0}^{T-1} S\left(q_{i}, v_{i}\right)-e v_{i},
$$

where the minimum is taken over all integers $T$ and all periodic sequence $\left(q_{i}, v_{i}\right) \in \mathcal{E}^{T}$, where $\mathcal{E}^{T}$ is the set defined in 4.6. The key point is that this set does not depend on $S$. The links between the averaged energy and the Birkhoff normal forms are now reduced to a straightforward computation in the integrable case, which leads to:

5.3. Lemma. - If $\ell: \mathbb{R}^{n} \rightarrow \mathbb{R}$ is a strongly convex function, and if $S(x, v)=\ell(v)$, then $\alpha^{S}$ is the Legendre transform of $\ell$.

5.4. LemmA. - If $S=\ell_{k}(v)+o_{k}(v)$, with a strongly convex $\ell_{k}$, then $\alpha^{S}(e)=$ $h_{k}(e)+o_{k}(e)$, where $h_{k}$ is the Legendre transform of $\ell_{k}$.

Proof. - One can chose functions $\ell^{+}$and $\ell^{-}: \mathbb{R}^{n} \rightarrow \mathbb{R}$ such that

$$
S^{-}(q, x)=\ell^{-}(v) \leqslant S(q, v) \leqslant \ell^{+}(v)=S^{+}(q, v)
$$

and such that $\ell^{+}-\ell^{-}=o_{k}$. By Lemma 5.3, the function $\alpha^{S^{ \pm}}$is the Legendre transform $h^{ \pm}$of $\ell^{ \pm}$. In view of 4.7 , we have $0 \leqslant h^{-}-h^{+} \leqslant o_{k}$. The proposition then follows from the fact that, by $5.2, h^{+}=\alpha^{S^{+}} \leqslant \alpha^{S} \leqslant \alpha^{S^{-}}=h^{-}$.

5.5. The proof of Theorems 1.9 and 1.10 now results from the following fact: For each simple neighborhood $U \subset U_{1}$ of $\mathcal{T}$, we have

$$
\alpha^{S}\left(\tau^{*} c\right)=\alpha_{U}(c)
$$

in a neighborhood of $0 \in H^{1}(\mathcal{T}, \mathbb{R})$. It follows that the germ of $\alpha^{S}$ does not depend of the choice of $S$, that the germ of $\alpha_{U}$ does not depend on $U$, and that these germs are equal:

$$
\alpha:=\alpha^{S} \circ \tau^{*}=\alpha_{U} .
$$


5.6. In order to prove this equality, let us consider a periodic orbit $X$ of $\phi$ contained in $U$. In view of 4.9 , we have $\mathcal{A}(X)-c[X] \geqslant m^{T}\left(\tau^{*} c\right)$. Since this holds for all periodic orbits, we conclude that, for each $c \in H^{1}(\mathcal{T}, \mathbb{R})$,

$$
\alpha_{U}(c) \leqslant \alpha^{S}\left(\tau^{*} c\right) .
$$

We will need the following non trivial proposition, proved in Section 6 for the other inequality.

5.7. Proposition. - Let $V$ be a simple neighborhood of $\mathbb{T}_{0}^{n}$. There exists a neighborhood $C$ of 0 in $\mathbb{R}^{n}$ such that, for each e $\in C$, there exists a sequence $Y_{k}$ of $T_{k}$-periodic e-minimal orbits of $F$ in $V$ such that

$$
\frac{H_{e}^{T_{k}}\left(Y_{k}\right)}{T_{k}}+\alpha^{S}(e) \longrightarrow 0 .
$$

5.8. Let $c \in H^{1}(\mathcal{T}, \mathbb{R})$ be such that $e=\tau^{*} c \in C$. Let $Y_{k}$ be the sequence of periodic orbits of $F$ given by 5.7. Since $Y_{k}$ is $e$-minimal, we have equality of the actions $\mathcal{A}\left(\tau\left(Y_{k}\right)\right)-e\left[Y_{k}\right]=H_{e}^{T_{k}}\left(Y_{k}\right)$. It follows that

$$
-\alpha_{U}(c) \leqslant \frac{\mathcal{A}\left(\tau\left(X_{k}\right)\right)-e\left[X_{k}\right]}{T_{k}} \longrightarrow-\alpha^{S}(e),
$$

hence $\alpha^{S} \circ \tau^{*}(c)=\alpha^{S}(e) \leqslant \alpha_{U}(c)$.

\section{Localization}

In this section, we prove Proposition 5.7. Let us note

$$
\widetilde{H}_{e}=H_{e}+\alpha^{S}(e) .
$$

We mention that $\widetilde{H}_{e}^{T}(Q) \geqslant 0$ if $Q \in E^{T}$.

6.1. Let us give a caracterisation of $\alpha^{S}$ :

$$
\liminf _{T \longrightarrow \infty}\left(m_{e}^{T}+T \alpha^{S}(e)\right)=0 .
$$

This is proved in $[\mathrm{Be}]$ and we shall reproduce the proof for the convenience of the reader. Let us first assume that there exists $T$ such that $m_{e}^{T}+T \alpha^{S}(e)=0$. Let $Q_{T}$ be a minimizing configuration of $H_{e}^{T}$ and $n * Q_{T}$ the $n T$-periodic configuration consisting of $n$ iterates of $Q_{T}$. We have $H_{e}^{n T}\left(n * Q_{T}\right)+n T \alpha(c)=0$ for any $n$, and so $\lim \inf \left(m_{e}^{T}+T \alpha^{S}(e)\right)=0$. Else assume that $m_{e}^{T}+T \alpha^{S}(e)>0$ for each $T$. By contradiction, let us assume in addition that $\liminf m_{e}^{T}+T \alpha^{S}(e)>0$, so that there is a real number $\ell$ with $m_{e}^{T}+T \alpha^{S}(e) \geqslant \ell>0$. Let us fix $r>0$ such that $\left|H_{e}\left(x_{0}, x\right)-H_{e}\left(x_{0}, x^{\prime}\right)\right| \leqslant \frac{1}{2} \ell$ for each $\left(x_{0}, x, x^{\prime}\right)$ such that $d\left(x, x^{\prime}\right) \leqslant 2 r$. There is a real number $p$ with $0<p<1$ such that, given $T$ points on the torus, there is a ball of radius $r$ containing at least $p T$ of them. Take a minimizing configuration $Q^{T}=\left(q_{i}^{T}\right)$ of $H_{e}^{T}$. After permutation of the indices, there are 
$K \geqslant p T$ points $q_{0}^{T}, q_{i_{1}}^{T}, \ldots, q_{i_{K-1}}^{T}$ contained in a ball of radius $r$. The configurations $Q_{k}=\left(q_{i_{k-1}}^{T}, q_{i_{k-1}+1}^{T}, \ldots, q_{i_{k}}^{T}\right)$ of length $T_{k}=i_{k}-i_{k-1}$ are almost periodic and we have

$$
H_{e}^{T_{k}}\left(Q_{k}\right)+T_{k} \alpha^{S}(e) \geqslant \frac{1}{2} \ell .
$$

Adding these inequalities for all $k$ we get

$$
H_{e}^{T}(Q)+T \alpha^{S}(e) \geqslant K \frac{1}{2} \ell \geqslant p T \frac{1}{2} \ell .
$$

This gives

$$
-\alpha^{S}(e) \leqslant \frac{H_{e}^{T}}{T}-\frac{1}{2} p \ell
$$

which is in contradiction with the definition of $\alpha^{S}$.

6.2. As a consequence, there exists a sequence $Y^{k}$ of $T_{k}$-periodic e-minimal orbits of $F$ such that $\widetilde{H}_{e}^{T_{k}}\left(Y_{k}\right) \rightarrow 0$. In order to prove Proposition 5.7, it is enough to see that this sequence is contained in $V$ if $e$ is small enough. This results from the following:

6.3. Lemma. - There exist a positive constant $\delta$ and a neighborhood $C$ of 0 in $\mathbb{R}^{n}$ such that, for each $e \in C$ and each e-minimal $T$-periodic orbit $X$ of $F$ not contained in $V$, we have $\widetilde{H}_{e}^{T}(X) \geqslant \delta$.

6.4. Lemma. - There exist a positive constant $\delta$ and a neighborhood $C$ of 0 in $\mathbb{R}^{n}$ such that, if $(q, p) \notin V$, then $S(q, V(q, p))+e V(q, p) \geqslant 2 \delta$ for each $e \in C$.

Proof. - We see from 5.4 that $\alpha^{S}(0)=0$ and $\left(\alpha^{S}\right)^{\prime}(0)=\omega$. Since $\alpha^{S}$ is convex, we have $\alpha^{S}(e) \geqslant e \omega \geqslant-\|e\| \cdot\|\omega\|$ hence

$$
\begin{aligned}
S(q, V(q, p))+e V(q, p) & \geqslant a\|V(q, p)-\omega\|^{2}-\|e\| \cdot\|V(q, p)\|+\alpha^{S}(e) \\
& \geqslant a\|V(q, p)-\omega\|^{2}-\|e\| \cdot\|V(q, p)-\omega\|-2\|e\| \cdot\|\omega\| .
\end{aligned}
$$

Since $G^{S}\left(\mathbb{T}_{0}^{n}\right)=\mathbb{T}^{n} \times\{\omega\}$, the norm $\|V(q, p)-\omega\|$ is bounded from below by a positive constant when $(q, p) \notin V$. The proof of the lemma is now straightforward.

6.5. Lemma. - If $C$ is small enough, then for each $e \in C$ and each configuration $q_{1}, \ldots, q_{T}$ (not necessarily periodic), we have $\widetilde{H}_{e}^{T}\left(q_{1}, \ldots, q_{T}\right) \geqslant-\delta$.

Proof. - As a first step, let us prove that, given two points $q, q^{\prime} \in \mathbb{T}^{n}$, there exists an integer $k$ and configuration $x_{0}=q, \ldots, x_{k}=q^{\prime}$ such that $\widetilde{H}_{e}^{k}\left(x_{0}, \ldots, x_{k}\right) \leqslant \delta$. Let us take any integer $k \in\left[\|e\|^{-1 / 2}, 2\|e\|^{-1 / 2}\right]$ (this interval contains integers when $e$ is small enough). Choose $\Delta \in \mathbb{R}^{n}$ such that $\|\Delta\| \leqslant n / k$ and $q^{\prime}=q+k(\omega+\Delta)$. Setting $x_{i}=q+i \Delta$, we have

$$
\widetilde{H}_{e}\left(x_{i}, x_{i+1}\right) \leqslant S\left(x_{i}, \omega+\Delta\right)+e(\omega+\Delta)+\alpha^{S}(e) \leqslant \frac{n b}{k^{2}}+\|e\|(1+3\|\omega\|)
$$

BULLETIN DE LA SOCIÉTÉ MATHÉMATIQUE DE FRANCE 
when $e$ is small enough. We have used that $\alpha^{S}(e) \leqslant 2\|\omega\| \cdot\|e\|$ when $e$ is small enough. We obtain

$$
\widetilde{H}_{e}^{k}\left(x_{1}, \ldots, x_{k}\right) \leqslant(n b+2(1+3\|\omega\|)) \sqrt{\|e\|} \leqslant \delta,
$$

when $e$ is small enough. Let us now consider a configuration $q_{0}, q_{1}, \ldots, q_{T}$. In view of the first step just proved, we can find an integer $k$ and a configuration $x_{0}=q_{T}, x_{1}, \ldots, x_{k}=q_{0}$ such that $\widetilde{H}_{e}^{k}\left(x_{0}, x_{1}, \ldots, x_{k}\right) \leqslant \delta$. The configuration

$$
Q=q_{0}, \ldots, q_{T}, x_{1}, \ldots, x_{k}
$$

is periodic hence

$$
0 \leqslant \widetilde{H}_{e}^{T+k}(Q) \leqslant \widetilde{H}_{e}^{T}\left(q_{1}, \ldots, q_{T}\right)+\delta
$$

which ends the proof.

6.6. We are in a position to end the proof of Lemma 6.3 hence of Proposition 5.7. Let $C$ and $\delta$ be chosen such that Lemma 6.4 and 6.5 hold. Let $X=\left(q_{i}, p_{i}\right)$ be a $T$-periodic orbit of $F$. Assume that $X$ is $e$-minimal and not contained in $V$. There exists $i$ such that $\left(q_{i}, p_{i}\right)$ is not in $V$, and we can as-

sume that $i=0$. It follows from 6.4 that $\widetilde{H}_{e}\left(q_{0}, q_{1}\right) \geqslant 2 \delta$. On the other hand, Lemma 6.5 imply that $\widetilde{H}_{e}^{T-1}\left(q_{1}, \ldots, q_{T}\right) \geqslant-\delta$, hence $\widetilde{H}_{e}^{T}(X) \geqslant \delta$.

\section{BIBLIOGRAPHY}

[Be] BernaRd (P.) - Homoclinic orbits to invariant sets of quasi-integrable exact maps, Ergod. Th. Dynam. Sys., t. 20 (2000), pp. 1583-1601.

[DS] Mc Duff (D.) \& SAlamon (D.) - Introduction to Symplectic Topology, Oxford Math. Monographs, 1995.

[He] HeRman (M.) - Inégalités a priori pour des tores lagrangiens invariants par des difféomorphismes symplectiques, Publ. Math. IHES, t. 70 (1989), pp. $47-101$.

[La] Lazutkin (V.) - KAM Theory and Semiclassical Approximations to Eigenfunctions, Springer, 1993.

[Ma] MAther (J.N.) - Action minimizing invariant measures for positive definite Lagrangian systems, Math. Z., t. 207 (1991), pp. 169-207.

[Si1] Siburg (K.F.) - Aubry-Mather theory and the inverse spectral problem for planar convex domains, Israel J. Math., t. 113 (1999), pp. 285-304.

[Si2] , Symplectic invariants of elliptic fixed points, Comment. Math. Helv., t. 75 (2000), pp. 681-700. 